\title{
ACOUSTIC EMISSION AND FORCE SENSOR FUSION FOR MONITORING THE CUTTING PROCESS
}

\author{
Erdal Emel and Elijah KanNatey-Asibu Jr \\ Department of Mechanical Engineering and Applied Mechanics, The University of Michigan, Ann Arbor, \\ MI 48109, U.S.A.
}

(Received 22 February 1988; and in revised form 28 September 1988)

\begin{abstract}
An acoustic emission and force-based sensor fusion system involving pattern recognition analysis has been used to detect tool breakage, chip form and a threshold level of tool flank wear in turning. When normalized with the resultant force, the force components in the cutting, radial and feed directions were found to be highly sensitive to variables such as feedrate, material hardness, tool coating and tool wear, depth of cut, and speed in fractional factorial experiments. A threedimensional analytical force model was extended to include the effect of flank wear in order to interpret the experimental findings. Subsequently, using an empirical bilinear relationship between the machining variables and forces, a filter was designed to eliminate the variable effects such that pattern recognition of tool failure under varying conditions was feasible. Results of the sensor fusion approach involving testing the system with the same data used in designing it when using AE and force signals indicate a $94 \%$ accuracy for sensing tool wear alone, whereas using only AE for detecting chip form and tool breakage indicate a 99 and $96 \%$ accuracy respectively.
\end{abstract}

\section{NOTATION}

a characteristic angle at tool nose

$C$ constant

$d$ depth of cut

$e$ filter output for a given force component

$f$ feedrate

$F$ force

F force feature vector

$\bar{F}$ normalized force

$K$ number of machining variables used in experimental design

$\left(l_{i} h_{n}\right.$ level of $i$ th variable at the $n$th test condition

$N$ number of factorial design test conditions

$r$ nose radius

$R$ resultant force

$v^{\prime}$ projection of wear land on tool face

$v_{B}$ wear land

$y_{i}$ magnitude of $i$ th variable

$\mathbf{Z}$ pattern vector

$\gamma_{\lambda}$ equivalent rake angle in the chip flow direction

$\lambda_{s}$ tool inclination angle

$\lambda_{\gamma \lambda}$ chip flow angle measured from $y$-axis

$\lambda_{y \gamma}$ angle of the equivalent cutting edge from $y$-axis

$\lambda$ chip flow angle with respect to equivalent cutting edge subscripts
$A$ major cutting edge
$B$ minor cutting edge
$C$ tangential direction
$F$ feed direction
$n$ test run number
$R$ radial direction

superscripts
tool face related quantities
" tool flank related quantities
$T$ transpose 


\section{INTRODUCTION}

Over four decades after Merchant's work [1], analysis of forces generated during machining is still a subject of research. However, force measurements have since been shown to be an invaluable output for monitoring the cutting process. The initial analysis based on orthogonal cutting was subsequently extended to oblique cutting by Stabler [2] and Colwell [3] and later, Mazur [4], Usui and Hirota [5] and Kirk et al. [6] used the geometrical rules developed for the chip flow direction to determine the forces on the tool face. The forces acting on the tool flank have also been studied by other researchers [7-10] and the conclusions indicate that the cutting forces in the tangential and feed directions increase with increasing flank wear land. Colwell et al. [10] used the ratio of feed force to cutting force and Koren [9] and Lan and Naerheim [11] used the cutting force itself in computer-based, online predictive algorithms for tool wear monitoring. Danai and Ulsoy [12] and Koren et al. $[13,14]$ investigated the feasibility of using force measurements to predict tool wear and concluded that it was necessary to include the effect of varying cutting conditions and consider the three orthogonal components of force for better performance. In an interesting work by Ulsoy and Han [15], a different approach was taken, and a multi-sensor strategy was proposed using cutting force, cutting force rate and acoustic emission (AE) count rate for detection of tool breakage.

In earlier work [16], we used AE for monitoring the turning process and achieved over $90 \%$ success in detecting tool breakage and chip segmentation, and over $85 \%$ success in identifying a threshold of flank wear under varying cutting conditions. These results were obtained when the classifier was tested with the same data used in designing it and are therefore optimistic.

In this paper, a combined sensor strategy is adopted using force and $\mathrm{AE}$ signals for improving the progressive wear detection performance as well as tool breakage monitoring, while using only AE signals for chip noise detection. The next section explains the sensor fusion approach. This is followed by an experimental design that forms the basis for a subsequent classification scheme.

\section{SENSOR FUSION}

Sensor fusion is an integrated sensing strategy designed to improve the performance and reliability of manufacturing process monitoring by utilizing various sensor outputs. Each of the sensors developed thus far has been found to be useful for a limited aspect of machine tool monitoring. Over the last three decades, various tool wear sensors were developed for machining but none of them has gained wide acceptance in application due to varying types of deficiencies inherent to each system. Among the in-process sensing techniques developed to date, a common problem has been the insufficient sensitivity of a sensor to a specific phenomenon of interest. For complete automation of machining processes, it is essential to monitor tool wear, tool breakage, as well as chip form. To enhance the capability of a sensor system for monitoring all the relevant aspects of the cutting process, we consider, in this paper, a multi-sensor strategy based on AE and force signals. The analysis emphasizes the sensitivity of force signals, since a similar sensitivity study has already been done for AE [16]. In that study, AE signals were shown to be related to the shearing, frictional and fracture processes during machining. While signals from fracture of chips and tools were highly repeatable, signals from tool wear related frictional processes showed more scatter which degraded the wear detection performance of the monitoring system. This degradation was explained by the stochastic variations resulting from the high sensitivity of AE to tool and workpiece material inhomogeneities coloring the wear related deterministic information in the signals.

To compensate for the deficiency, we are using force signals for augmenting the original AE based tool monitoring system. The results indicate that when structural vibrational effects are filtered, the three force components provide reliable information about tool wear.

A mechanistic model showing the tool geometry dependency of feed, radial and tangential components of cutting force is presented in Appendices A, B and C. The analysis is intended to establish a basis for the signal processing approach explained later in the paper. The 
signal processing approach consists of a linear discriminant function based pattern recognition of signals which is preceded by filtering to eliminate the effects of controlled cutting variables. The filtering is accomplished using a model of sensor outputs obtained from experimental data.

\section{SENSOR OUTPUT MODELING}

In general, forces generated during machining are affected by various process variables. Due to the large number of variables involved, an experimental study to analyse the effect of each variable one at a time would ordinarily be expensive. Selection of a two-level fractional factorial experimental design reduces the necessary number of tests to obtain the prevailing relationships between the input variables, $y_{i}$ (i.e. cutting speed, feedrate, depth of cut, material hardness, insert tool type, tool wear), and the output variables, $F$ (i.e. cutting forces). The details of the experimental design were presented in an earlier paper [16] and are reproduced in Table 1 (Appendix D), but the output variables in that case were the power spectral components of the AE signal. A summary of the selected variables are the test conditions is given in Appendix D. Actually, AE and force measurements were made simultaneously during the experiments.

With force measurements obtained by the fractional factorial design, the individual effect of the $i$ th process variable, $y_{i}$, on any force component, $F$, can be determined [16] as

$$
\Delta F_{y_{i}}=\frac{2}{N} \sum_{n=1}^{N}\left(l_{i}\right)_{n} F_{n} \quad i=1, \ldots K .
$$

In general, an experimental design at two levels yields only first-order interaction effect of variables (i.e. bilinear functional relationship), whereas the strength of interaction of more than two variables is rarely found significant. Here, we only consider the first-order interaction effect of two variables, $y_{i}$ and $y_{j}$, on force components $F$, and is given by [16]

$$
\Delta F_{y_{i} y_{j}}=\frac{2}{N} \sum_{n=1}^{N}\left(l_{i}\right)_{n}\left(l_{j}\right)_{n} F_{n} \quad i=1, \ldots K-1 ; j=i+1, \ldots K .
$$

Equations (1) and (2) provide a quantitative basis for an empirical relationship to predict the force levels at any point within the operational range of each process variable. For any given process output, the general form of such an equation for $K$ variables is given in [16] as

$$
\hat{F}=F_{0}+\sum_{i=1}^{K}\left(y_{i}-y_{i(0)}\right) \frac{\Delta F_{y_{i}}}{\Delta y_{i}}+\frac{1}{2 !} \sum_{i=1}^{K-1} \sum_{j=i+1}^{K}\left(y_{i}-y_{i(0)}\right)\left(y_{j}-y_{j(0)}\right) \frac{4 \Delta F_{y_{i} y_{j}}}{\Delta y_{i} \Delta y_{j}} \text {. }
$$

Among the six variables given in Table 2 (Appendix D), the only one that is qualitative by definition is the cutting tool type, the levels of which may be symbolically represented by $(-1)$ and $(+1)$ in equation (3). The other variables may take on any value within their operational limits.

\section{PATTERN CLASSIFIER}

The process variables in machining have been classified as controlled (cutting speed, feedrate, depth of cut, material hardness and tool grade) and disturbance (flank and crater wear, tool breakage, chip segmentation) type [16]. However, in the analysis of forces, only tool wear and breakage are considered as disturbance inputs, since chip segmentation is unobservable by force measurements. Due to the three-dimensional nature of oblique cutting, wear and fracture phenomena can have varying effects on the components of force in the cutting, feed and radial directions. Thus, measurement of force components will generally show a diversity of patterns and magnitudes.

For this reason, we use a pattern classification scheme for characterizing the various failure modes. Furthermore, to eliminate absolute magnitude effects, the individual components are normalized with respect to the resultant force:

$$
\begin{gathered}
\bar{F}_{C}=\frac{F_{C}}{R}, \quad \bar{F}_{R}=\frac{F_{R}}{R}, \quad \bar{F}_{F}=\frac{F_{F}}{R}, \\
R=\sqrt{F_{C}^{2}+F_{R}^{2}+F_{F}^{2}} .
\end{gathered}
$$


Each normalized component is taken as a feature of the force pattern. Therefore, the process monitoring problem becomes one of pattern recognition, assuming all the force features for a given phenomenon as clustered around a distinct mean value with minimum variance and enough separation between the means of each phenomenon. The basis for this pattern recognition approach is explained in earlier articles on $\mathrm{AE}[17,18]$. A linear discriminant function based pattern recognition system requires a feature vector as an input. Here, the features are the three normalized force components along with the resultant force, and represented as

$$
\mathbf{F}^{T}=\left\{\begin{array}{llll}
\bar{F}_{C} & \bar{F}_{R} & \bar{F}_{F} & R
\end{array}\right\}
$$

A pattern vector $\mathbf{Z}$ is further defined by

$$
\mathbf{Z}=\left\{\begin{array}{c}
\mathbf{F} \\
-1
\end{array}\right\}
$$

Pattern classification based on normalized forces may successfully detect disturbance inputs due to tool wear and breakage provided that the controllable inputs do not vary. However, with many machining operations, cutting conditions frequently change. If the effects of such controlled input variations can be predicted using equation (3), then their contribution may easily be eliminated from the measured forces so that the classification scheme be made insensitive to such variations.

The effects of cutting speed, feedrate, depth of cut, tool and workpiece material are eliminated by filtering [16], i.e. subtracting the output of equation (3), $\hat{f}$, from the plant output, $f$, which may be any force feature in the vector $\mathbf{F}$ generated by the actual process:

$$
e=F-\hat{F} \text {. }
$$

With the exception of tool wear, levels of all other inputs are the same in the plant and the observer given by equation (3), and tool wear is assumed to be maximum at the observer (i.e. $y_{1}=y_{1}(+)$ ) during the entire period of process monitoring. Thus, the filter output, $e$, should be zero when the tool is worn, i.e.

$$
e=F-\hat{F} \approx 0
$$

but for a sharp tool or breakage

$$
e=F-\hat{F} \neq 0 \text {. }
$$

A new feature vector, $\mathbf{E}$, is then obtained using the error, $e$, for each feature in $\mathbf{F}$ and to replace $\mathbf{F}$ in $\mathbf{Z}$ in equation (4). The new feature vector is designated by

$$
\mathbf{E}^{T}=\left\{e_{\bar{F}_{r}} e_{\bar{F}_{R}} e_{\bar{F}_{F}} e_{R}\right\}
$$

which is used in linear discriminant functions designed to identify tool wear and breakage under varying cutting conditions.

\section{EXPERIMENTAL}

The experimental set-up and conditions are essentially the same as described in [16] except for the force data acquisition system which is outlined in the following paragraphs.

Force measurements were made using a three component piezoelectric Kistler dynamometer, model 9257A, attached to an MTGNL-16-4D (ISO MTGNL-25-25-M-12) tool holder. An AE piezoelectric transducer was also attached to the tool holder for simultaneous AE signal detection. The dynamometer assembly had a resonant frequency at approximately $4 \mathrm{kHz}$, and thus, according to the manufacturer's recommendations, $0.3 \times 4=1.2 \mathrm{kHz}$ was considered the maximum reliable frequency range of force signals. The three charge outputs of the dynamometer were converted to voltage signals using Kistler Dual Mode charge amplifiers, model 5004, and further recorded on a Racal tape recorder, model 7DS, for offline analysis. The three force components were digitized along with a pulse signal using a 12bit analog to digital converter, model ADV11-C, and a PDP-11/23 minicomputer. The pulse 
train was for synchronizing the force signals with the AE signal which was sampled separately with a different set-up. A sampling frequency of $2 \mathrm{kHz}$ was used and an analog filter, Krohn-Hite model 3320 was set for low-pass filtering with a cut-off frequency of $1 \mathrm{kHz}$ before sampling, to prevent aliasing.

To improve synchronization, for each continuous type of AE signal of $1 \mathrm{~ms}$ duration, 10 samples of force measurements within 5 ms duration were averaged to obtain a single corresponding value.

Since only tool fracture generates transient force signals, for a corresponding AE signal of $1 \mathrm{~ms}$ duration, a single maximum value of force in the cutting direction was recorded along with the corresponding feed and radial forces at the moment of fracture. Another set of tool fracture samples, 29 in all, were obtained at varying feedrates of $0.010-0.024 \mathrm{ipr}$ $(0.254-0.609 \mathrm{~mm} / \mathrm{rev})$, but with common depth of cut of $0.063 \mathrm{in} .(1.600 \mathrm{~mm})$, cutting speed of $600 \mathrm{fpm}(3.051 \mathrm{~m} / \mathrm{s})$ and material hardness of $360 \mathrm{BHN}$, using two types of tool material, VC7 $(7 \% \mathrm{Co}, 10 \% \mathrm{TiC}, 2 \% \mathrm{PaC}, 82 \% \mathrm{WC}$ by weight) and $\mathrm{VN} 8(\mathrm{Co}, \mathrm{TaC}, \mathrm{TiC}$, in WC base and coated with TiN).

Ten averaged force values were obtained from each of the 32 test conditions to give a mean output, $F_{n}$. It is essential to know that although the sharp tool samples are homogenous in sharp tool definition, the worn tool samples represent a combination of various flank and crater formations, but at least with a common base of $0.012 \mathrm{in} .(0.3048 \mathrm{~mm})$ flank wear.

\section{RESULTS AND DISCUSSION}

Force measurements in the cutting, radial and feed directions for test 13 in Table 1 are shown in Fig. 1, for a $15 \mathrm{~s}$ duration pass. The test begins with a sharp tool and ends with $0.020 \mathrm{in} .(0.508 \mathrm{~mm})$ nose wear and significant crater wear. The cutting conditions used for this test were such that the resulting wear rate was very high. However, a number of the other tests resulted in light cutting conditions where wear was observed after minutes of cutting. The overall results obtained therefore represent a variety of tool wear modes. The sensitivity of the cutting force, $F_{C}$, to progressive wear is observed to be much less than that of the radial, $F_{R}$, and feed, $F_{F}$, forces. Increasing nose wear causes a change in the ratio of forces when compared to the initial conditions.

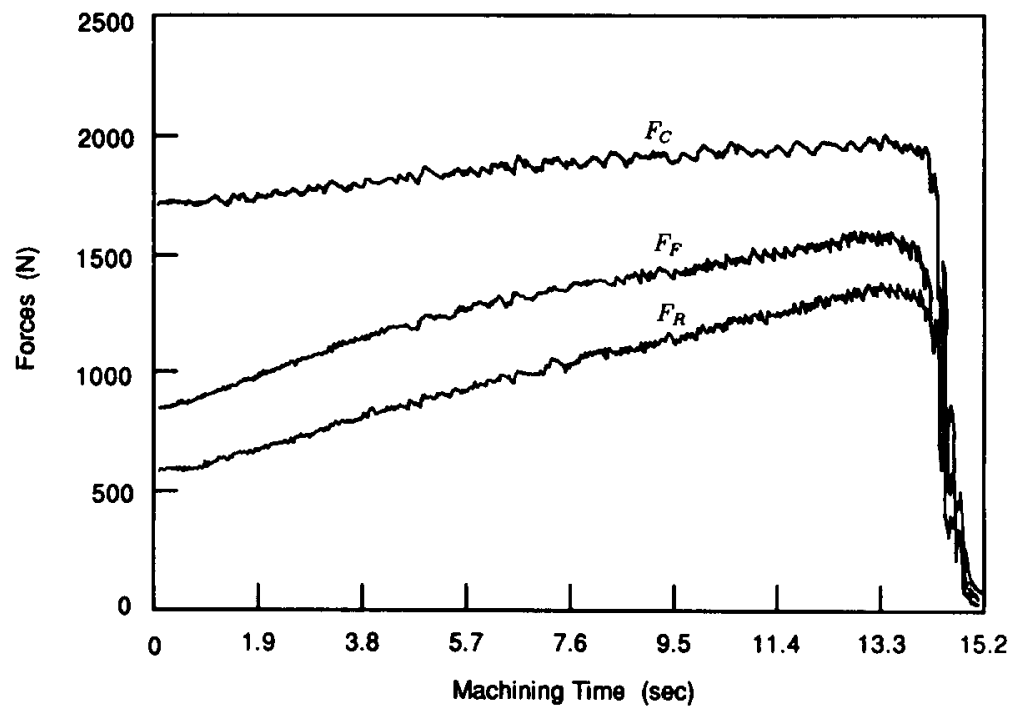

FIG. 1. Three-dimensional force measurements (Test 13) 


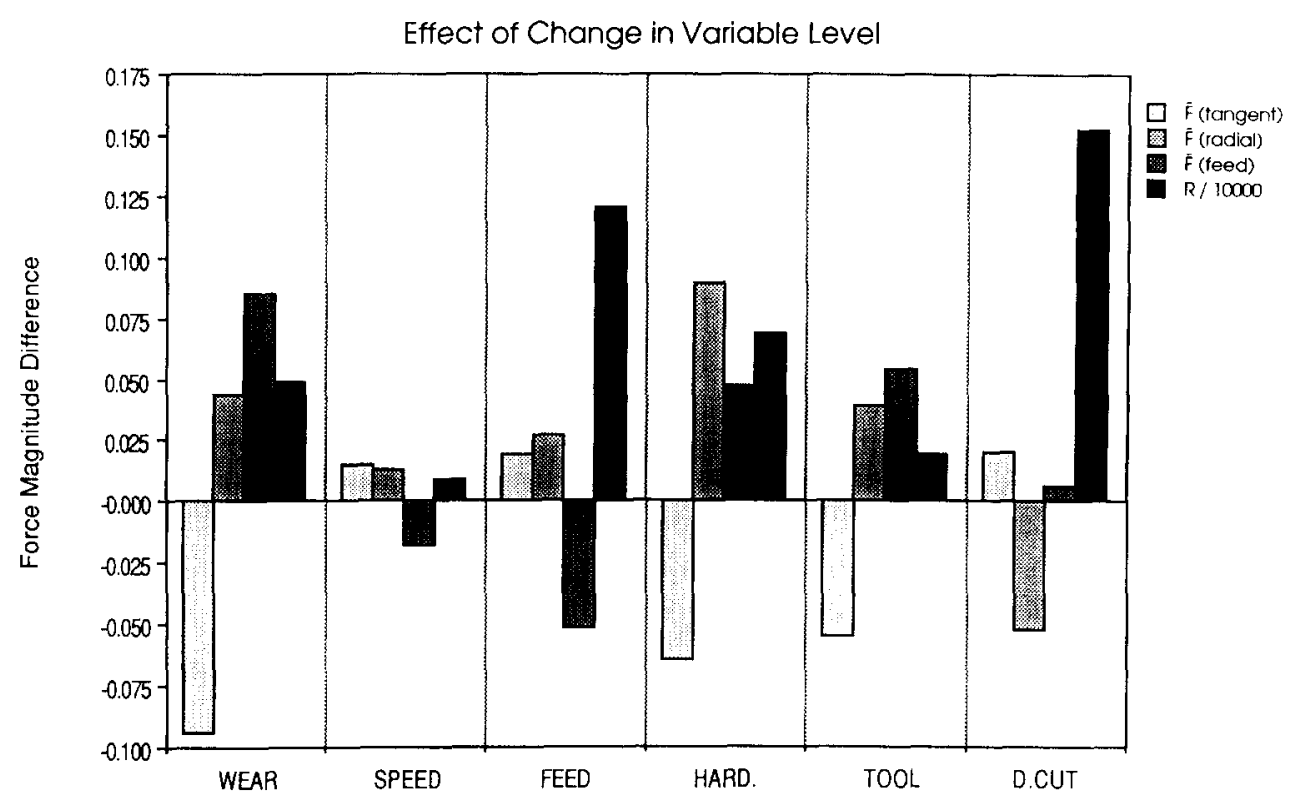

Fig. 2. Individual effects of variables on forces, $\Delta F_{s i}$.

Each of the 32 test conditions was run with a new cutting edge, and from each run, ten measurements (samples) were made. Using these measurements, the individual effects of input variables on normalized and resultant force components, as given by equation (1), are shown in Fig. 2. When the flank wear level is increased from 0.001 to $0.012 \mathrm{in}$. $(0.0254-0.3048 \mathrm{~mm})$, the effects on normalized forces are an increase in the feed and radial components and a decrease in the cutting component, with a significant increase in the resultant force. Increasing the cutting speed from 525 to $735 \mathrm{fpm}(2.67-3.73 \mathrm{~m} / \mathrm{s})$, does not result in appreciable effects on normalized and resultant forces. With the feedrate increased from 0.008 to $0.016 \mathrm{ipr}(0.2032-0.4064 \mathrm{~mm} / \mathrm{rev})$, a decrease in normalized feed force is seen despite a large increase in the resultant force. When material hardness is increased from 255 to $370 \mathrm{BHN}$, the effects on normalized feed, radial, and resultant forces are in the increasing direction although the cutting force decreases. Changing the uncoated carbide insert grade, VC7 to a coated carbide grade, VN8, implicitly results in a change from crater-dominated wear to flank-dominated wear, and the corresponding effects on normalized forces are similar to the wear effect. For the depth of cut, a change from 0.063 to $0.124 \mathrm{in}$. $(1.6-3.1496 \mathrm{~mm})$, effects a decrease in the normalized radial force.

Overall, all variables except the cutting speed seem to have considerable effect on the forces. For the resultant force, all the input variables result in increasing output. Feedrate and depth of cut effects appear to show the maximum values even though the corresponding normalized force variations were not as significant.

From the experimental measurements and equations (1) and (2), an empirical model, i.e. equation (3), was obtained, and in addition, a mechanistic force model was developed (Appendices A, B and C) to explain the experimental design results. Although not intended as an exhaustive analysis, a quantitative comparison of the empirical model with the experimental measurements is given in Table 3 (Appendix D) for selected test conditions where the tools were allowed to wear, although they were originally sharp. For a given test. some of the measurements were made at the beginning, and others at the end.

The results of the empirical model represented by equation (3) show reasonably close correlation with measured values (Table 3 ) which can also be qualitatively verified by the mechanistic model. This indicates that equation (3) can be used as a filter to improve tool failure detection under varying conditions. 


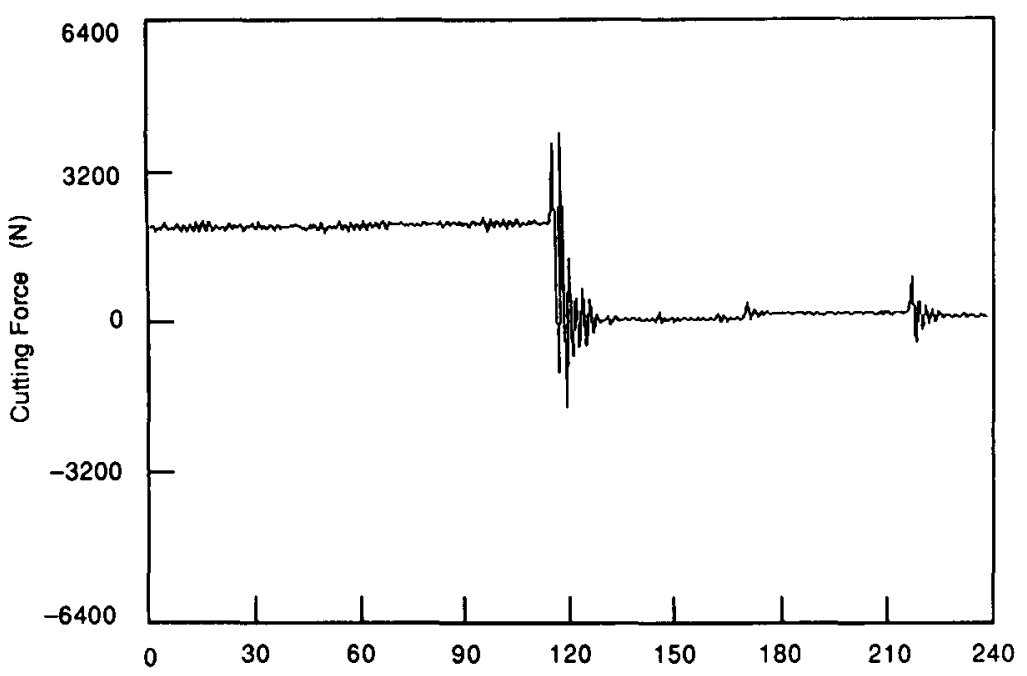

(a)

Machining Time (msec)

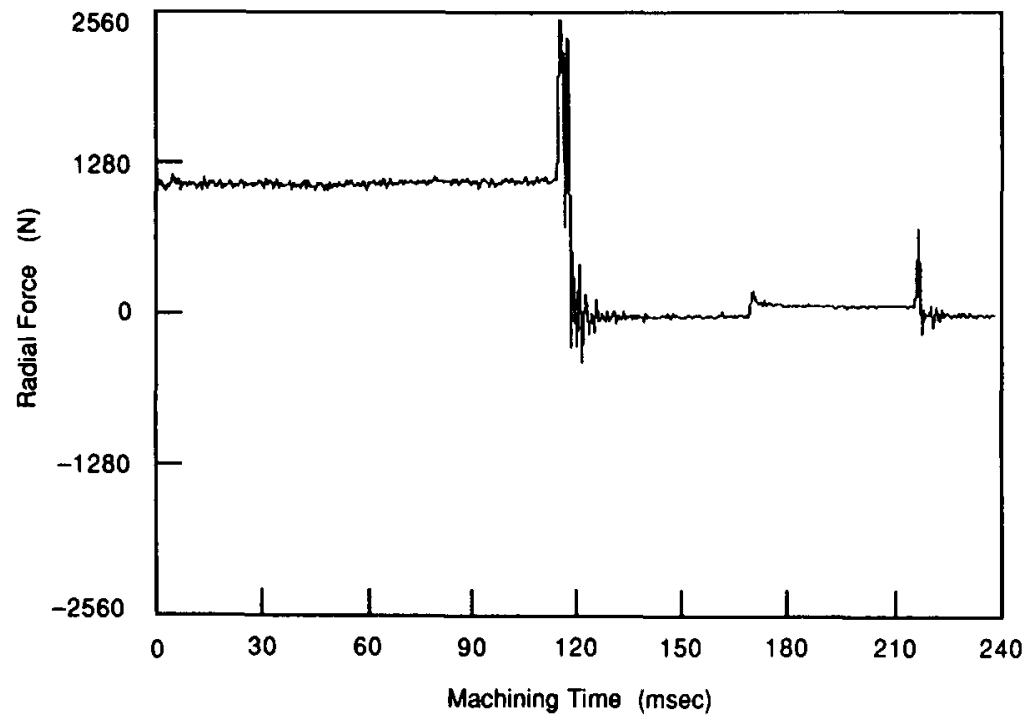

(b)

Machining Time (msec)

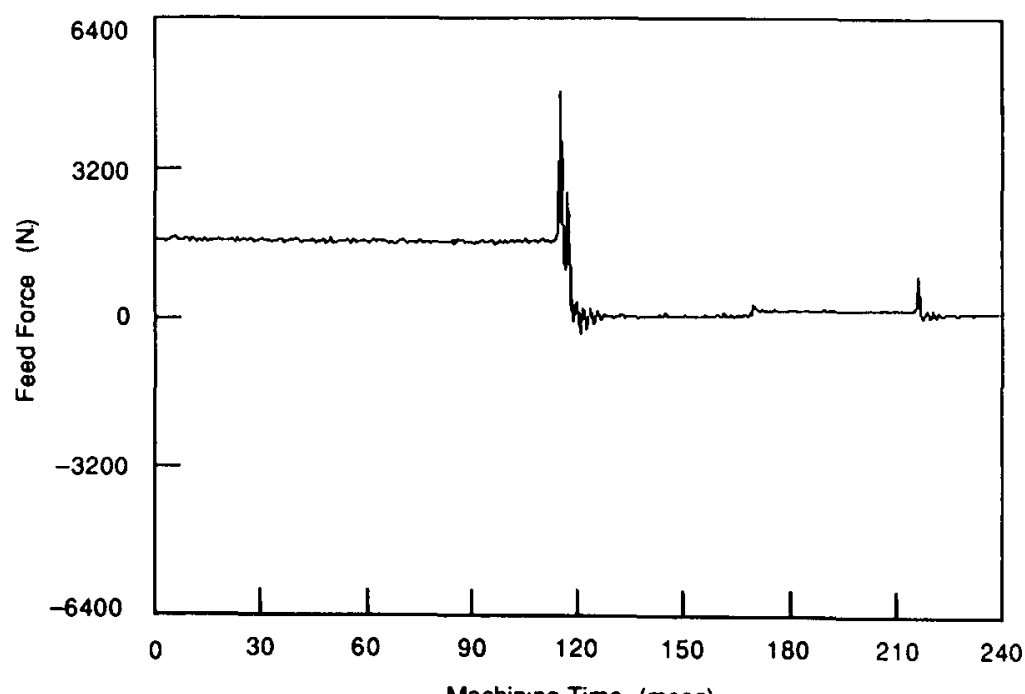

(c)

Machinıng Time (msec)

FIG. 3. Force measurements during tool fracture. (a) Cutting force. (b) Radial force. (c) Feed force. 
The behavior of force components during a typical tool fracture event are shown in Fig. 3. It is evident that the individual components exhibit different characteristics, which when combined will enable the fracture phenomenon to be easily identified.

The results of pattern recognition analysis of 160 samples of sharp tool and 120 samples of worn tool signals are given in Table 4 (Appendix D), for mixed cutting conditions. The classification performance for both sharp and worn tool classes, obtained prior to and after filtering are designated by columns $\mathbf{F}$ and $\mathbf{E}$, respectively. Using the filtering approach with resubstitution and leave-one-out testing methods [20], sharp tool recognition was improved from 85 to $100 \%$, under varying cutting conditions. Similarly, worn tool detection was improved from 66 to $100 \%$.

By adding 29 tool breakage samples to the sharp tool and worn tool data sets, a simultaneous classification of three classes can be made and the results are shown in Table 5 (Appendix D). On average, when filtering is used sharp tool and tool breakage recognition drastically improve to $100 \%$ for each case. However, worn tool performance is not as good as desired. Moreover, chip noise is not detectable by force signals. Thus, since AE has been found to be highly sensitive to chip noise and tool breakage [16] a sensor fusion or combined sensor scheme involving $\mathrm{AE}$ and force signals was considered appropriate for further improving the performance. On this basis, we use the hierarchical decision tree strategy illustrated in Fig. 4 for monitoring the machining process by first using acoustic emission to determine if the process is generating continuous or transient signals. At this stage, $94 \%$ of the continuous and $99 \%$ of the transient signals can be correctly identified [16]. Subsequently, force signals are used to discriminate the continuous process being generated either by sharp or worn tools, with a $100 \%$ accuracy. If the process is found to be transient, then using the acoustic emission signals again, it can be identified as being either chip noise or tool breakage with 100 and $97 \%$ accuracy, respectively [16]. The results obtained, however, are rather optimistic, since they resulted from testing the sensor system using the same data used in designing it. Due to the considerable expense involved in tool wear tests, "independent" results obtained by testing the system with a completely new set of data will be the subject of subsequent publications. In any case, the results thus far demonstrate the feasibility of incorporating pattern recognition analysis and sensor fusion of $\mathrm{AE}$ and force for monitoring the cutting process.

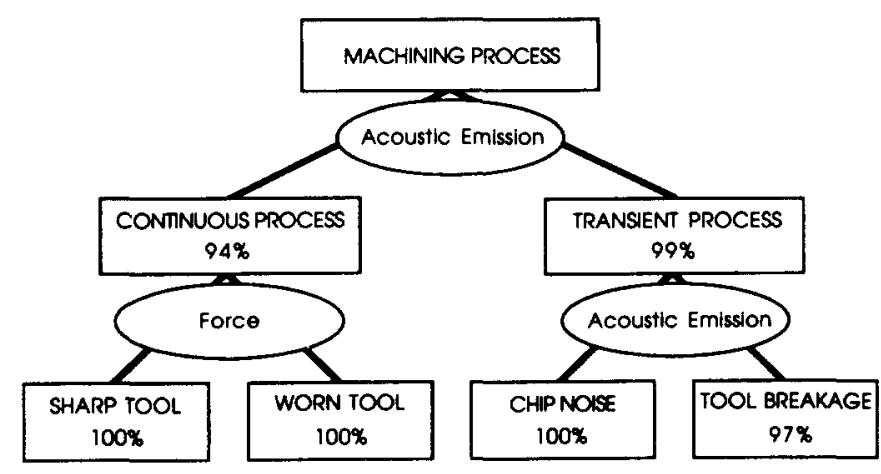

Fug. 4. Decision strategy for sensor fusion and classification performance for tool monitoring. 


\section{CONCLUSIONS}

In a continuing effort to improve tool failure detection, $\mathrm{AE}$ and force signals have been combined in a sensor fusion strategy.

To determine the effect of cutting parameters as well as flank wear on force generation during machining, a mechanistic model of orthogonal forces acting on the tool face and flank was first analysed. Then, based on a fractional factorial design, experiments were conducted to substantiate the effect of variables and further derive an empirical relationship. Normalizing the resultant force enhanced the geometrical dependency of orthogonal forces, thus generating force patterns highly sensitive to wear and fracture phenomena.

The results of the experiments while machining 4340 steel at two hardness levels with coated and uncoated carbides at various cutting conditions qualitatively agreed with the mechanistic model. Although the effect of crater wear was unaccounted for, the mechanistic model was found to be quite useful in explaining the nature of experimental findings and establishing a physical base for the empirical relationship.

Therefore the empirical force relationship, being sufficiently accurate, was used as a filter to eliminate the effect of controlled cutting conditions, improving the tool wear detection by pattern recognition to almost perfection. A combination of $\mathrm{AE}$ and force features, as a sensor fusion, was then simultaneously used in the same classifier for identification of the machining process.

Acknowledgements-The support of this paper by the University of Michigan Industrial Consortium on Diagnostic Sensing, the Industrial Technology Institute, and the National Science Foundation under Grant No. DMC 8607198 is gratefully acknowledged.

\section{REFERENCES}

1. M. E. MERCHANT, Basic mechanics of the metal cutting process. ASME J. appl. Mech. 11, 168 (1944).

2. G. V. Stabler, The fundamental geometry of cutting tools. Proc. I.M.E. 165, 14 (1951).

3. L. V. COLwELL, Predicting the angle of chip flow sor single-point cutting tools. Trans. ASME 76, 199 (1954).

4. J. C. MAZUR, A technique to optimize control of process variable in related studies of causes and effects of metal cutting behaviour. Ann. CIRP 15, 287 (1967).

5. E. UsUI and A. HIROTA, Analytical prediction of three dimensional cutting process-Part 2. Chip formation and cutting force with conventional single-point tool. ASME J. Engng Ind. 100, 229 (1978).

6. J. A. KIRK, D. K. ANAND and C. MCKINDRA, Matrix representation and prediction of three-dimensional cutting. ASME J. Engng Ind. 99, 828 (1977).

7. N. N. Zorev, Metal Cutting Mechanics. Pergamon Press, Oxford (1966).

8. E. G. Thomsen, A. G. MacDonald and S. Kobayashi, Flank friction studies with carbide tools reveal sublayer plastic flow. ASME J. Engng Ind. 75, 53 (1962).

9. Y. KOREN, Flank wear model of cutting tools using control theory. ASME J. Engng Ind. 100, 103 (1978).

10. L. V. Colwell, J. C. Mazur and W. R. DeVries, Analytical strategies for automatic tracking of tool wear. Proc. 6th North American Manufacturing Conf., p. 276 (1978).

11. M. S. LAN and Y. NAERHEIM, In-process detection of tool breakage in milling. ASME J. Engng Ind. 108, 191 (1986).

12. K. Danal and G. UlsoY, A dynamic state model for on-line tool wear estimation in turning. ASME Publ., PED 18, $137(1985)$.

13. Y. KOREN, A. G. UlsoY and K. DanaI, Tool wear and breakage detection using a process model. Ann. CIRP 35(1), 283 (1986)

14. Y. Koren, K. Danai, A. G. Ulsoy and T. R. Ko, Monitoring tool wear through force measurement. Proc. 15th North American Manufacturing Research Conf., Lehigh University, Bethlehem (1987).

15. A. G. ULSOY and E. HAN, Tool breakage detection using a multi-sensor strategy. Proc. IF AC/IFORS Symp. Control Science and Technology for Development, Beijing, China, p. 181 (1985).

16. E. EMEL and E. KanNATEY-AsjBu $\mathbf{J}_{\mathbf{R}}$, Acoustic emission monitoring of the cutting process - negating the influence of varying conditions. Submitted to ASME J. Engng Ind. (1987).

17. E. KANNATEY-ASIBU $J_{R}$, On the application of the pattern recognition method to manufacturing process monitoring. Proc, 10th NAMRC, Hamilton, Ontario, p. 487 (1982).

18. E. KANNATEY-ASIBU JR and E. EMEL, Linear discriminant function analysis of acoustic emission signals for cutting tool monitoring. J. Mech. Syst. Signal Process. 1(4), 333 (1987).

19. E. EMEL and E. KANNATEY-AsJBU JR, Tool failure monitoring in turning by pattern recognition analysis of AE signals. ASME J. Engng Ind. 110, 137 (1988).

20. E. EMfl and E. KanNATEY-Asibu JR, Characterization of tool wear and breakage by pattern recognition analysis of acoustic emission signals. Proc. 14th North American Manufacturing Research Conf., University of Minnesota, Minneapolis, p. 266 (1986). 


\section{APPENDIX A: FORCES IN OBLIQUE CUTTING}

After Stabler's derivation of the chip flow rule [2], Colwell adapted it to three-dimensional cutting [3]. A brief review of Colwell's approach is given in Appendix B, and is illustrated in Fig. A1a, where the critical variables are the chip flow angle, $\lambda_{\gamma \lambda}$, and the equivalent rake angle in the chip flow direction, $\gamma_{\lambda}$. In determining $\lambda_{\gamma \lambda}$ and $\gamma_{\lambda}$, an equivalent cutting edge, the line connecting the two extreme corners A and B of the chip load (Fig. A la and b) is assumed and that reduces the three-dimensional configuration into one of two dimensions across the imaginary edge, $\overline{A B}$. Inclination of the tool on its major and minor cutting edges causes the chip flow direction to deviate from the line normal to the equivalent cutting edge to an angle, $\lambda$, on the tool reference plane. It has been shown by Mazur [4] and Usui and Hirota [5] that an assumption of orthogonal cutting along the equivalent cutting edge is reasonable.

(a)
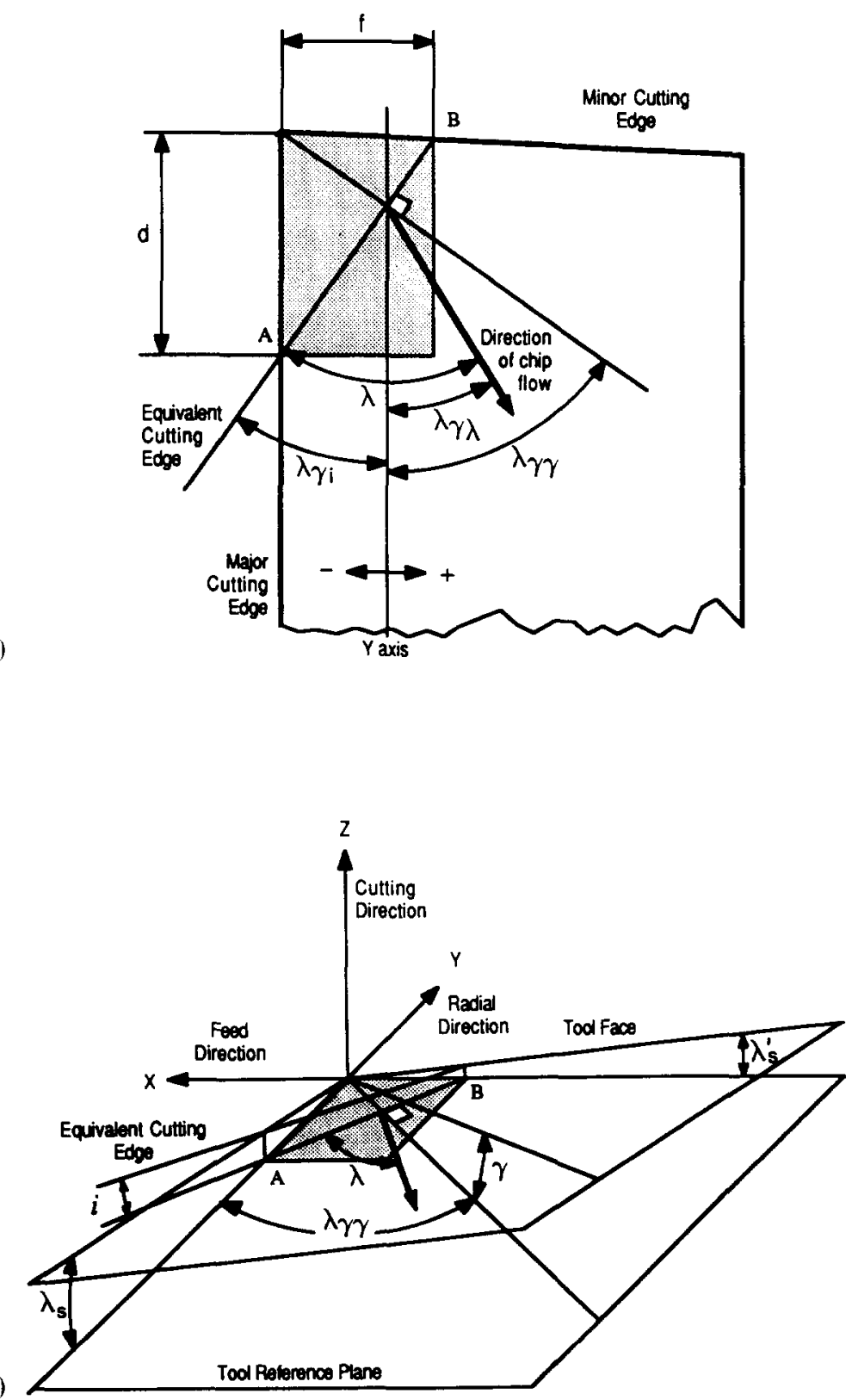

FIG. A1. (a) Chip flow direction projected on tool reference plane. (b) Three-dimensional view of tool face. 


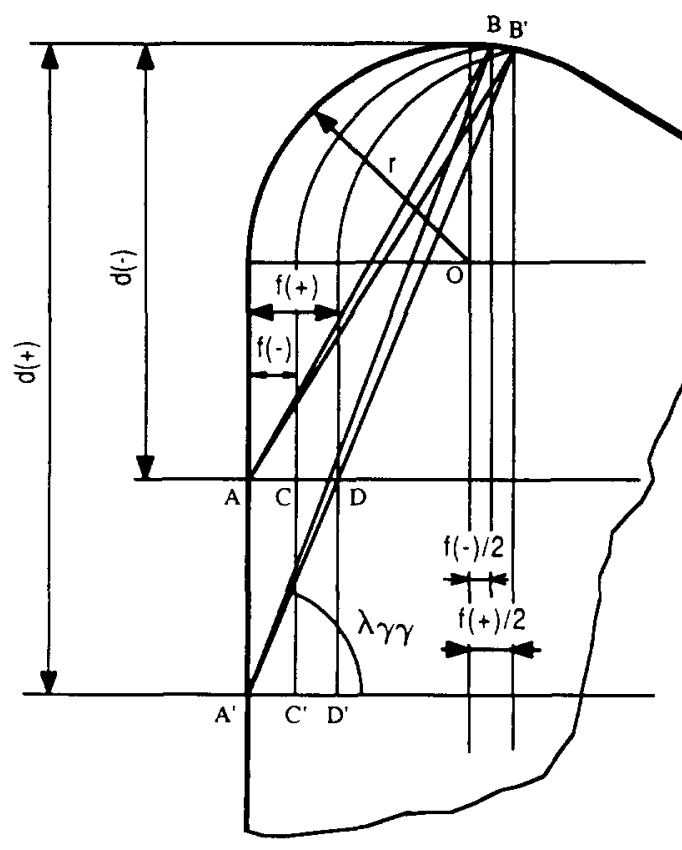

(a)

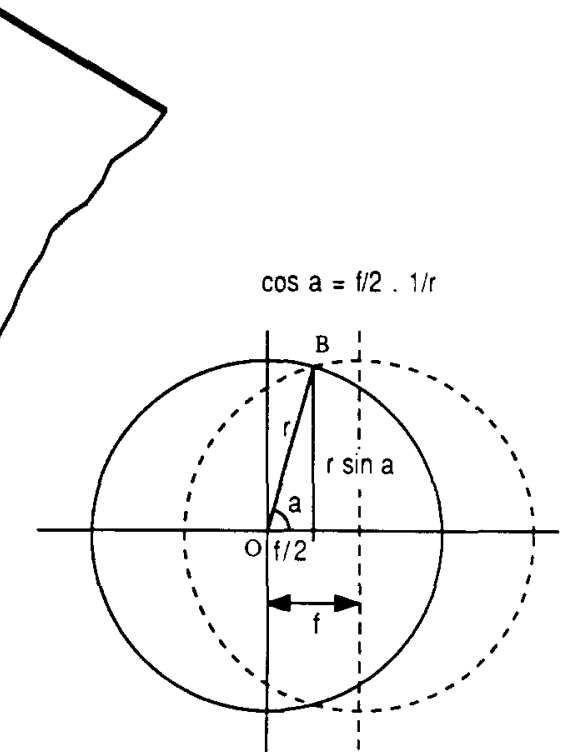

(b)

FiG. A2. Orientation of the equivalent cutting edge. (a) Varying feed and depth of cut effect.

(b) Geometrical relationship at tool nose.

Here, we extend Colwell's approach [3] by further considering a tool geometry with a nose radius and flank wear. In Fig. A2a, a TNMA type insert is schematically shown at one of its corners on the tool reference plane. Since the angle, $\lambda_{y}$, of the equivalent cutting edge, depends on the geometry of chip load, we can relate $\lambda_{\gamma \gamma}$ directly to the feed, $f$, depth of cut, $d$, and nose radius, $r$. At a given depth of cut, $d(-)$, changing the feed from $f(-)$ to $f(+)$ rotates the equivalent cutting edge from $\overline{A B}$ to $\overline{A B^{\prime}}$. Similarly, at a depth of cut of $d(+)$, changing feed from $f(-)$ to $f(+)$ changes the orientation of the edge from $\overline{A^{\prime} B}$ to $\overline{A^{\prime} B^{\prime}}$. Using the geometrical relationship for angle $a$, i.e. $a=\arccos [f /(2 r)]$, in Fig. A2b, the angular orientation of the equivalent cutting edge is defined by

$$
\tan \hat{\lambda}_{\gamma \gamma}=\frac{d-r(1-\sin a)}{r+f / 2} .
$$

In Fig. A3a, the effect of flank wear on the equivalent cutting edge is shown, with the line $\overline{A B}$ becoming $\overline{A^{\prime} B^{\prime \prime}}$ for a flank wear land of $v_{B}$. Thus the initial chip load under the area $\overline{A B C}$ becomes $\overline{A^{\prime} B^{\prime} B^{\prime \prime} C^{\prime}}$. For a given wear land of $v_{B}$, there will be a loss of tool material of $v_{B}^{\prime}$ in the radial direction and from the geometrical relationship for angle $a$, i.e. $a=\arcsin \left[\left(r-v_{B}^{\prime}\right) / r\right]$, in Fig. A3b, the corresponding equivalent edge orientation, $\lambda_{\gamma \gamma}$, will be

$$
\tan \lambda_{\gamma \gamma}=\frac{d-v_{B}^{\prime}}{r(1+\cos a)-v_{A}^{\prime}} .
$$

Replacing equation (B.1) with equations (A.1) and (A.2), an improved estimate of the chip flow angle, $\lambda_{y \lambda}$, and the equivalent rake angle, $\gamma_{\lambda}$, can be obtained from equation (B.8) and equation (B.9), and subsequently used to determine the forces acting on the sharp or worn tools, as the case may be.

Determination of the force components acting on a sharp tool as given by Colwell et al. [10], is presented in Appendix C (Fig. A4a) whereas the force components acting under the existence of flank wear are shown in Fig. A4b. For simplification, we consider the normal force acting on the tool flank to be given [9] by

$$
F_{N}^{\prime \prime}=C v_{B} \text {. }
$$

$F_{N}^{\prime \prime}$ acts in the plane containing the feed and radial directions, and can be resolved into the components, $F_{R}^{\prime \prime}$ and $F_{F}^{\prime \prime}$, depending on the angular orientation of the flank wear land, $\lambda_{y}$, (Fig. A4b) which is approximated to be equal to the chip flow direction, $\lambda_{y \lambda}$. Thus, the radial and feed components are given by:

$$
\begin{aligned}
& F_{R}^{\prime \prime}=F_{N}^{\prime \prime} \cos \lambda_{\gamma} \approx C v_{B} \cos \lambda_{y \lambda} \\
& F_{F}^{\prime \prime}=F_{N}^{\prime \prime} \sin \lambda_{\gamma} \approx C v_{B} \sin \lambda_{y \lambda} .
\end{aligned}
$$




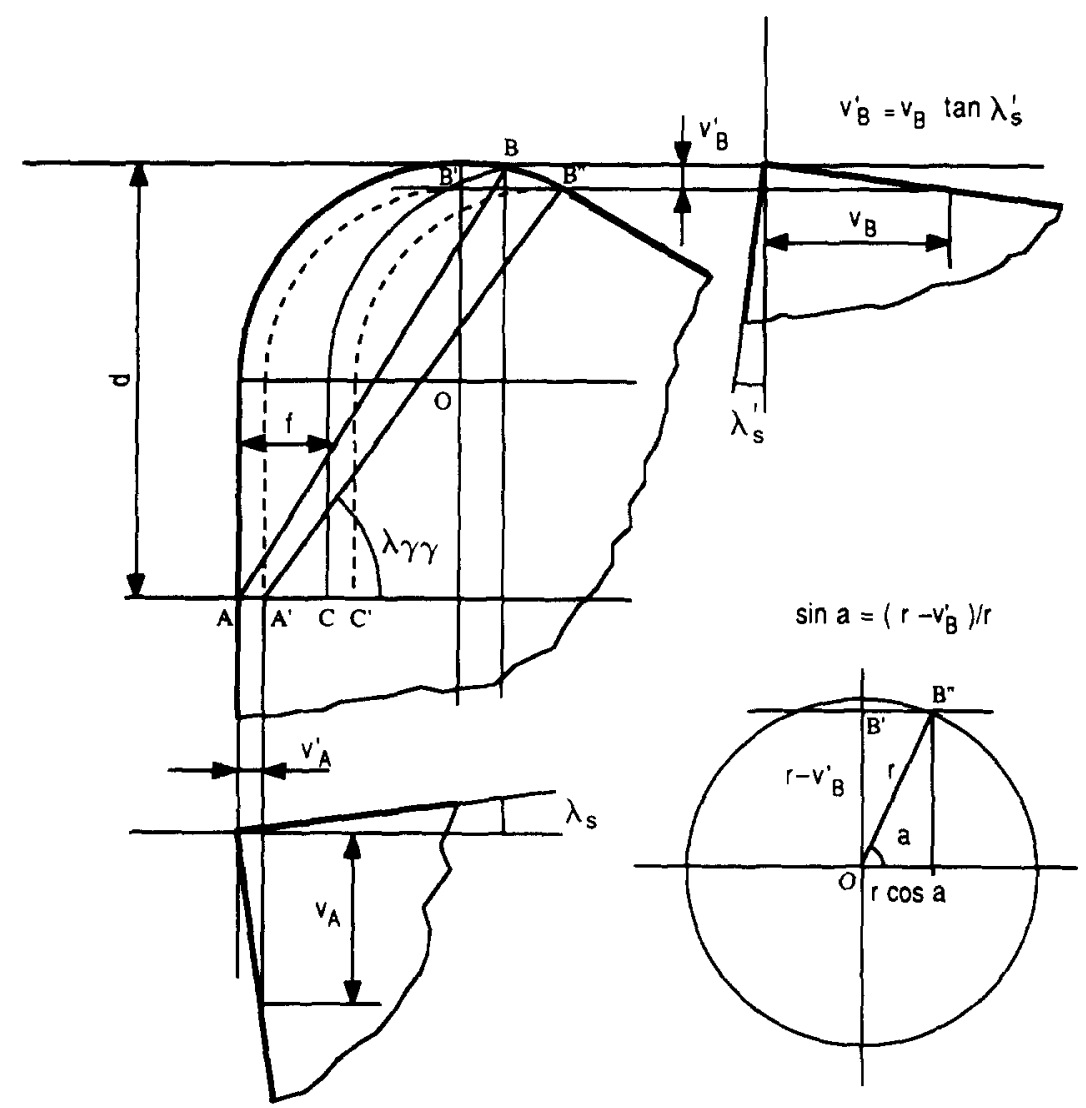

(a)

(b)

FIG. A3. Orientation of the equivalent cutting edge. (a) Flank wear effect. (b) Geometrical relationship at tool nose.

The component of the resultant force, $R^{\prime \prime}$, on the tool flank in the cutting direction is determined by the friction angle, $\beta^{\prime \prime}$, between the tool and the newly formed surface of the workpiece.

$$
F_{C}^{\prime \prime}=F_{N}^{\prime \prime} \tan \beta^{\prime \prime} \approx C v_{B} \tan \beta^{\prime \prime} \text {. }
$$

The friction angle, $\beta^{\prime \prime}$, is expected to be different from the tool face friction angle, $\beta^{\prime}$, since they depend on different boundary conditions of the tool face and flank. Finally, the resultant forces in the orthogonal directions are obtained as the summation of forces acting upon the tool face and flank, namely,

$$
\begin{aligned}
& F_{C}=F_{C}^{\prime}+F_{C}^{\prime \prime} \\
& F_{R}=F_{R}^{\prime}+F_{R}^{\prime \prime} \\
& F_{F}=F_{F}^{\prime}+F_{F}^{\prime \prime} .
\end{aligned}
$$



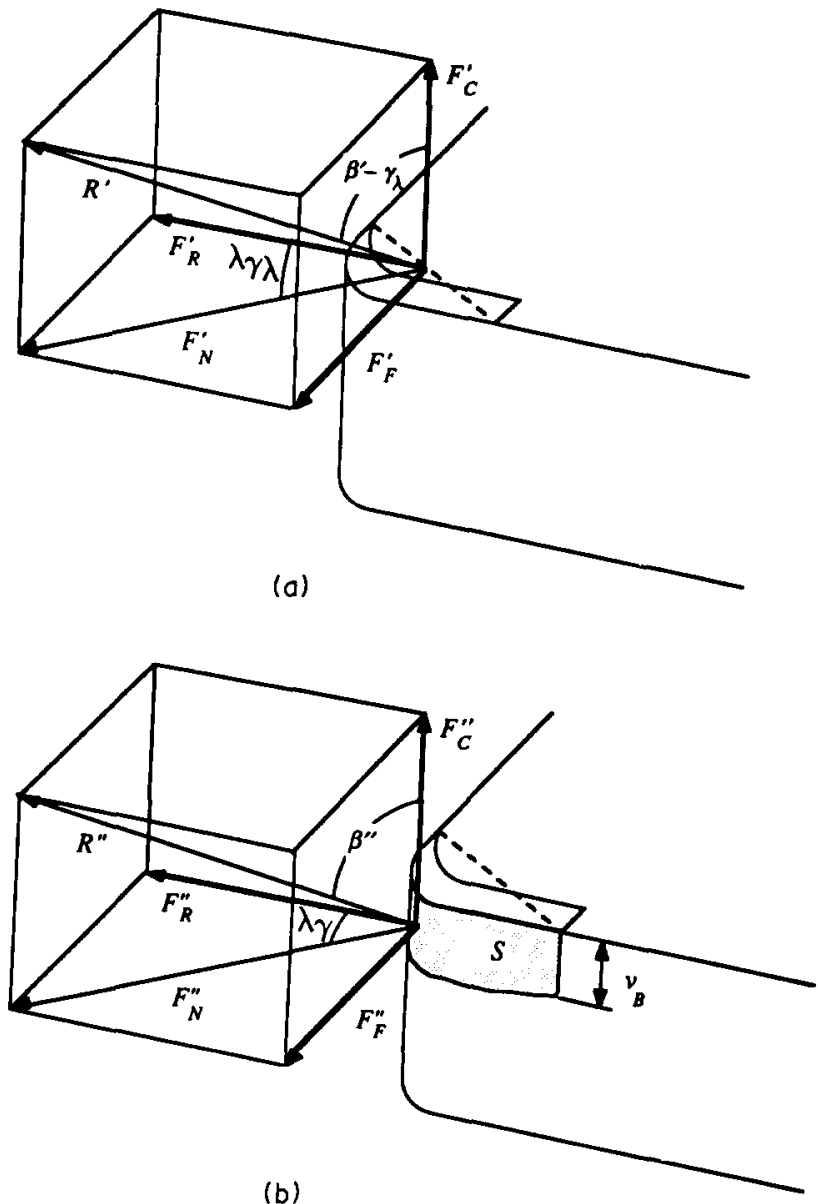

FIG. A4. Orthogonal forces acting on cutting tool. (a) Forces on tool face. (b) Forces on tool flank.

\section{APPENDIX B: DETERMINATION OF CHIP FLOW DIRECTION}

In Fig. Ala and b, the orientation of the equivalent cutting edge [3]:

$$
\begin{aligned}
\tan \lambda_{\gamma \gamma} & =\frac{d}{f} \\
\lambda_{\gamma i} & =-\left|90^{\circ}-\lambda_{\gamma \gamma}\right| .
\end{aligned}
$$

Slope of any line on the tool face (Fig. Alb):

$$
\tan \gamma=\sin \lambda_{\gamma \gamma} \tan \lambda_{s}^{\prime}+\cos \lambda_{y \gamma} \tan \lambda_{s} .
$$

Angle of inclination of equivalent cutting edge (Fig. A1b):

$$
\tan i=\sin \lambda_{\gamma i} \tan \dot{\lambda}_{s}^{\prime}+\cos \lambda_{\gamma i} \tan \lambda_{s}
$$

Slope of tool face in direction normal to the equivalent cutting edge:

$$
\tan \omega=\tan \gamma \cos i \text {. }
$$

Chip flow angle with respect to the equivalent cutting edge:

$$
\begin{gathered}
\cot \lambda=\frac{\sin i}{\cos \omega}-\tan \gamma \frac{\sin 2 i}{2} \\
\lambda=180^{\circ}-\left|\dot{\lambda}_{\gamma}\right| .
\end{gathered}
$$


Chip flow angle (Fig. A lb):

$$
\lambda_{, i}=\lambda_{i}-\left|i_{i i}\right|
$$

Effective rake angle in the chip flow direction:

$$
\tan {\gamma_{\lambda}}_{\lambda}=\sin \lambda_{j \lambda} \tan \dot{\lambda}_{s}^{\prime}+\cos \lambda_{y \lambda} \tan \lambda_{s}
$$

\section{APPENDIX C: ORTHOGONAL FORCES ON TOOL FACE}

In Fig. A4a, the force component in the cutting direction [10]:

$$
F_{c}^{\prime}=\frac{\tau_{s} A_{c}}{\sin \phi \cos \left(\beta^{\prime}-\gamma_{\lambda}\right)}
$$

where $\phi$ is the shear angle, $\beta^{\prime}$ is the friction angle on tool face, $A_{c}$ is the area of chip load and $\tau_{s}$ is the shear stress. Projection of the resultant force, $R^{\prime}$ on to the tool face yields the normal, radial and feed force components:

$$
\begin{gathered}
F_{N}^{\prime}=F_{C}^{\prime} \tan \left(\beta^{\prime}-\gamma_{\lambda}\right) \\
F_{R}^{\prime}=F_{C}^{\prime} \tan \left(\beta^{\prime}-\gamma_{\lambda}\right) \cos \lambda_{\gamma \lambda} \\
F_{F}^{\prime}=F_{C}^{\prime} \tan \left(\beta^{\prime}-\gamma_{\lambda}\right) \sin \lambda_{\gamma \lambda} .
\end{gathered}
$$

\begin{tabular}{|c|c|c|c|c|c|c|c|}
\hline \multirow{2}{*}{$\begin{array}{l}\text { Test } \\
\text { No. }\end{array}$} & \multicolumn{6}{|c|}{ Level of variables } & \multirow{2}{*}{$\frac{\text { Output }}{F_{n}}$} \\
\hline & $l_{1}$ & $l_{2}$ & $l_{3}$ & $l_{4}$ & $l_{5}$ & $l_{6}$ & \\
\hline 1 & - & - & $\ldots$ & - & - & - & $F_{1}$ \\
\hline 2 & + & - & - & - & - & + & $F_{2}$ \\
\hline 3 & $\ldots$ & + & $\ldots$ & - & - & + & $F_{3}$ \\
\hline 4 & + & + & - & - & - & - & $F_{4}$ \\
\hline 5 & $\ldots$ & - & + & - & - & + & $F_{5}$ \\
\hline 6 & + & - & + & - & - & - & $F_{6}$ \\
\hline 7 & - & + & + & - & - & - & $F_{7}$ \\
\hline 8 & + & + & + & - & - & + & $F_{\mathrm{B}}$ \\
\hline 9 & - & - & - & + & - & + & $F_{9}$ \\
\hline 10 & + & - & $\ldots$ & + & - & - & $F_{10}$ \\
\hline 11 & - & + & - & + & - & - & $F_{11}$ \\
\hline 12 & + & + & $\ldots$ & + & - & + & $F_{12}$ \\
\hline 13 & - & - & + & + & - & - & $F_{13}$ \\
\hline 14 & + & - & + & + & - & + & $F_{14}$ \\
\hline 15 & - & + & + & + & - & + & $F_{15}$ \\
\hline 16 & + &.+ & + & + & - & - & $F_{1 \text { h }}$ \\
\hline 17 & - & $\ldots$ & - & $\ldots$ & + & + & $F_{1}$, \\
\hline 18 & + & - & - & - & + & - & $F_{18}$ \\
\hline 19 & - & + & - & & + & & $F_{19}$ \\
\hline 20 & + & + & - & - & + & + & $F_{20}$ \\
\hline 21 & - & $\ldots$ & + & $\ldots$ & + & $\ldots$ & $r_{21}$ \\
\hline 22 & + & - & + & - & + & + & $F_{22}$ \\
\hline 23 & $\ldots$ & + & + & - & + & + & $F_{23}$ \\
\hline 24 & + & + & + & - & + & - & $F_{24}$ \\
\hline 25 & - & - & - & + & + & - & $F_{25}$ \\
\hline 26 & + & - & & + & + & + & $r_{20}$ \\
\hline 27 & - & + & $\ldots$ & + & + & + & $r_{27}$ \\
\hline 28 & + & + & $\ldots$ & + & + & $\ldots$ & $F_{2 B}$ \\
\hline 29 & - & - & + & + & + & + & $F_{29}$ \\
\hline 30 & + & - & + & + & + & - & $F_{30}$ \\
\hline 31 & - & + & + & + & + & - & $r_{31}$ \\
\hline 32 & + & + & + & + & + & + & $r_{32}$ \\
\hline
\end{tabular}

APPENDIX D: FRACTIONAL FACTORIAL EXPERIMENTAL DESIGN

TablF 1. Fractional factorial design OF experiments 
TABLE 2. EXPERIMENTAL CONDITIONS

\begin{tabular}{|c|c|c|c|c|}
\hline \multirow{2}{*}{\multicolumn{2}{|c|}{ Variable }} & \multicolumn{2}{|c|}{ Level of variable } & \multirow[t]{2}{*}{ Units } \\
\hline & & $(-)$ & $(+)$ & \\
\hline$y_{1}$ & $\begin{array}{l}\text { Tool flank } \\
\text { wear }\end{array}$ & $\begin{array}{c}0.001 \\
(0.0254)\end{array}$ & $\begin{array}{c}0.012 \\
(0.3048)\end{array}$ & $\begin{array}{l}\text { in. } \\
(\mathrm{mm})\end{array}$ \\
\hline$y_{2}$ & Cutting speed & $\begin{array}{c}525 \\
(2.67)\end{array}$ & $\begin{array}{c}735 \\
(3.73)\end{array}$ & $\begin{array}{l}\text { sfpm } \\
(\mathrm{m} / \mathrm{s})\end{array}$ \\
\hline$y_{3}$ & Feed rate & $\begin{array}{c}0.008 \\
(0.2032)\end{array}$ & $\begin{array}{c}0.016 \\
(0.4064)\end{array}$ & $\begin{array}{c}\mathrm{ipr} \\
(\mathrm{mm} / \mathrm{rev})\end{array}$ \\
\hline$y_{4}$ & $\begin{array}{l}\text { Material } \\
\text { hardness }\end{array}$ & 255 & 370 & BHN \\
\hline$y_{5}$ & $\begin{array}{l}\text { Cutting tool } \\
\text { (TNMA 432) } \\
\text { (ISO THMA 22-04-08) }\end{array}$ & $\mathrm{VC7}$ & VN8 & \\
\hline$y_{6}$ & Depth of cut & $\begin{array}{c}0.063 \\
(1.6000)\end{array}$ & $\begin{array}{c}0.124 \\
(3.1496)\end{array}$ & $\begin{array}{l}\text { in. } \\
(\mathrm{mm})\end{array}$ \\
\hline
\end{tabular}

TABLE 3. COMPARISON OF MEASURED FORCES WITH EMPIRICAL MODEL

\begin{tabular}{crrrrrr}
\hline & \multicolumn{3}{c}{ Measured value } & \multicolumn{3}{c}{ Empirical model } \\
Test & \multicolumn{1}{c}{$F_{C}$} & \multicolumn{1}{c}{$F_{R}$} & \multicolumn{1}{c}{$F_{F}$} & \multicolumn{1}{c}{$F_{C}$} & $F_{R}$ & $F_{F}$ \\
\hline Sharp 10 & 993 & 336 & 571 & 950 & 325 & 591 \\
Worn 10 & 1174 & 545 & 960 & 1153 & 523 & 960 \\
Sharp 11 & 940 & 311 & 518 & 854 & 292 & 556 \\
Worn 11 & 1134 & 508 & 809 & 1135 & 496 & 888 \\
Sharp 13 & 1753 & 619 & 995 & 1817 & 634 & 989 \\
Worn 13 & 1960 & 1190 & 1533 & 1954 & 975 & 1686 \\
\hline
\end{tabular}

Table 4. Performance of Sharp tool-worn TOOL CLASSIFICATION

\begin{tabular}{lllll}
\hline & \multicolumn{4}{c}{ Force output } \\
Testing method & \multicolumn{2}{c}{ F } & \multicolumn{2}{c}{ E } \\
\hline Resubstitution & 86 & 67 & 100 & 100 \\
Leave-one-out & 84 & 66 & 100 & 100 \\
Average & 85 & 66 & 100 & 100 \\
\hline
\end{tabular}

Table 5. Performance of Sharp tool worn tool tool BREAKAGE CLASSIFICATION

\begin{tabular}{lllllll}
\hline & \multicolumn{5}{c}{ Force output } \\
Testing method & & F & & \multicolumn{3}{c}{ E } \\
\hline Resubstitution & 82 & 52 & 93 & 100 & 85 & 100 \\
Leave-one-out & 77 & 51 & 93 & 100 & 84 & 100 \\
Average & 79 & 51 & 93 & 100 & 84 & 100 \\
\hline
\end{tabular}

